\title{
Efficacy of Combination Chemo- Immunotherapy as a First-Line Treatment for Advanced Non-Small- Cell Lung Cancer Patients With HER2 Alterations: A Case Series
}

\section{OPEN ACCESS}

Edited by:

Herbert Yu,

University of Hawaii,

United States

Reviewed by:

Gregory Alan Otterson,

The Ohio State University,

United States

Laura Bonanno,

Veneto Institute of Oncology

(IRCCS), Italy

*Correspondence:

Ke Wang

wang2ke@126.com

Yalun Li

lunlunhx@qq.com

Specialty section:

This article was submitted to

Thoracic Oncology,

a section of the journal

Frontiers in Oncology

Received: 25 November 2020 Accepted: 26 March 2021 Published: 20 April 2021

Citation:

Zhao S, Xian X, Tian P, Li W Wang $K$ and Li Y (2021) Efficacy of Combination Chemo-immunotherapy as a First-Line Treatment for Advanced Non-Small-Cell Lung Cancer Patients With HER2 Alterations: A Case Series.

Front. Oncol. 11:633522.

doi: 10.3389/fonc.2021.633522

\author{
Shuang Zhao ${ }^{1}$, Xinghong Xian ${ }^{2}$, Panwen Tian ${ }^{1}$, Weimin $\mathrm{Li}^{1}, \mathrm{Ke} \mathrm{Wang}^{1 *}$ and Yalun $\mathrm{Li}^{1 *}$ \\ ${ }^{1}$ Department of Respiratory and Critical Care Medicine, West China Hospital, Sichuan University, Chengdu, China, \\ ${ }^{2}$ Clinical Medicine of West China Medical School/West China Hospital, Sichuan University, Chengdu, China
}

Objective: Although the treatment of non-small-cell lung cancer (NSCLC) patients with human epidermal growth factor receptor 2 (HER2) alterations has been studied for years, the overall response rate (ORR) of these patients is still unsatisfactory, and more therapeutic strategies are needed. Little is known about the combination of chemoand immunotherapy in HER2-altered lung cancer treatment.

Materials and Methods: We report five cases of advanced NSCLC with HER2 insertion mutation or amplification treated with immunotherapy combined with chemotherapy as the first-line treatment. The HER2 alteration type, duration of treatment and survival were also analyzed.

Results: The five advanced NSCLC patients, three with HER2 mutations and two with HER2 amplifications, received chemo-immunotherapy as the first-line treatment. The average patient age was 54.6 years. Three patients were females, and two were males. Among all the patients, only one had a smoking history. The immunotherapies used were as follows: two patients were treated with sintilimab, and three patients were treated with pembrolizumab. Only one patient had squamous carcinoma, and she was also the only patient with a complete response (CR). The progression-free survival (PFS) ranged from 2-12 months, with a median PFS of 8.0 months.

Conclusions: Chemo-immunotherapy may be a promising first-line treatment option for NSCLC patients with HER2 alterations. Further clinical trials are required to confirm this therapeutic option.

Keywords: human epidermal growth factor receptor2, immunotherapy, chemotherapy, non-small-cell lung cancer, prognosis 


\section{INTRODUCTION}

Lung cancer is known as one of the deadliest cancers worldwide and causes more deaths than prostate, breast, brain and colorectal cancers combined (1). Non-small-cell lung cancer (NSCLC) comprises approximately $85 \%$ of all lung cancer cases (2). Human epidermal growth factor 2 (HER2) is a rare oncogenic driver that is altered in $1 \%$ to $3 \%$ of NSCLC patients (3). The main types of HER2 alterations in lung cancer include gene insertion mutation, gene amplification and protein overexpression (4). Chemotherapy remains an important component of treatment for HER2-altered NSCLC patients, although HER2 positive tumors are relatively insensitive to chemoradiotherapy $(5,6)$. Several HER2-targeted tyrosine kinase inhibitors (TKIs) and antibodies have also been tested for the treatment of these patients. However, the overall response rate (ORR) was unsatisfactory, at only $7.4 \%$ for HER2-targeted TKIs such as neratinib, lapatinib and afatinib (7). More therapeutic strategies for NSCLC patients with HER2 alterations are needed. Little is known about the combination of immunotherapy and chemotherapy in the treatment of lung cancer with HER2 alterations. Therefore, we described five advanced NSCLC cases with HER2 mutation or amplification and immunotherapy combined with chemotherapy as the firstline treatment. We hope this case series will provide new clinical therapeutic insight for this class of patients.

\section{CASE PRESENTATION}

From January 2019 to June 2020, five patients with advanced NSCLC with HER2 alterations and chemo-immunotherapy as the first-line treatment were admitted to the Lung Cancer Center, West China Hospital, Sichuan University. High-throughput next-generation sequencing (NGS) technology was used to assess the presence and type of HER2 alterations in the biopsy specimens of all patients. The status of PD-L1 was also tested by immunohistochemistry. This retrospective study was approved by the Committee on Medical Ethics of West China Hospital, Sichuan University.

\section{Patients With HER2 Mutation}

Three patients harbored HER2 insertion mutations.

The first patient (Case 1) was a 37-year-old Asian female who was a never-smoker and had a symptom of severe headache. She was finally diagnosed with left lung adenocarcinoma with brain, bone, hilar and mediastinal lymph node metastases (cT2N2M1c, stage IVB). She harbored a HER2 insertion mutation in exon 20 (p. A775_G776insYVMA). EGFR, ALK, ROS-1 and PD-L1 testing was performed, and none of these targets were expressed. She received chemotherapy (carboplatin and pemetrexed) and sintilimab for 6 cycles with a partial response $(\mathrm{PR})$ as her best response. Then, she experienced progressive disease (PD) with new brain metastases and was treated with pyrotinib, a pan-ErbB receptor TKI, for 2 months. However, she continued to progress with multiple new brain metastases and died because of a hemorrhagic cerebral hernia.

The second patient (Case 2) was a 65-year-old Asian man with a 10-pack-year smoking history who was diagnosed with cT4N3M1c stage IVB lung adenocarcinoma by pleural effusion smear and cytological examination. He complained of cough for 4 months. The NGS panel revealed a HER2 mutation (exon 20, p. A775_G776insYVMA) without concurrent alterations or PD-L1 expression. He was treated with carboplatin and pemetrexed combined with sintilimab for 4 cycles. His best response was stable disease (SD). Then, he experienced progressive malignant pleural effusion. One month after starting anlotinib, a chest CT scan showed a reduction in pleural effusion. The patient maintained SD until his last visit.

The third patient (Case 3) was a 52-year-old Asian man who was a never-smoker and developed cough and bloody sputum for 2 months. Chest CT showed a left lower lung mass with multiple bilateral pulmonary nodules. Brain MRI and bone single photon emission CT (SPECT) were all negative. Through percutaneous lung biopsy and left supraclavicular lymph node biopsy, he was finally diagnosed with cT4N3M1c stage IVB right lung adenocarcinoma. A HER2 mutation (exon 20, p. A775_G776insYVMA) was found from his initial molecular testing, but no other gene alterations were identified. $\mathrm{He}$ received carboplatin and pemetrexed chemotherapy and pembrolizumab for 2 cycles. Unfortunately, he experienced rapid progression within 2 months. He was then treated with docetaxel, carboplatin and bevacizumab for 1 month. He is currently enrolled in an EGFR/HER2-targeted TKI clinical trial (DZD9008).

\section{Patients With HER2 Amplification}

There were two patients with HER2 amplification.

The fourth patient (Case 4) was a 72-year-old Asian female never-smoker who complained of dorsalgia for 9 months. She was diagnosed with lung adenocarcinoma by percutaneous lung biopsy. CT showed metastatic mediastinal lymphadenopathy, SPECT scanning showed multiple bone metastases, and MRI showed evidence of brain metastases. The clinical stage was cT1N2M1c stage IVB. She was found to have HER2 amplification (copy number:2.6) without other gene alterations or PD-L1 expression. She was treated with carboplatin, pemetrexed, and pembrolizumab for 9 cycles. Then, she progressed with multiple new liver and bone metastases after 12 months and started on docetaxel and pembrolizumab with radiographic evidence of $\mathrm{SD}$, which was sustained for 4.0 months up to the study endpoint.

The fifth patient (Case 5) was a 47-year-old Asian female never-smoker who had no symptoms. Her chest CT scan showed multiple pulmonary nodules. She was diagnosed with cT4N0M1a stage IVA lung squamous carcinoma by percutaneous lung biopsy. NGS testing of her lung biopsy specimen was performed, and it showed HER2 amplification (copy number:3.22). EGFR, ALK, ROS-1 and PD-L1 expression were all negative. She received chemotherapy (carboplatin and pemetrexed) and pembrolizumab for 4 cycles followed by 
pembrolizumab maintenance therapy, with a complete response (CR) as her best response. The patient was still in remission at the endpoint of this study.

\section{Summary of Patients}

The average patient age was 54.6 years. Three patients were females, and two were males. Among all the patients, only one had a smoking history (Case 2). The HER2 mutation type, treatments, responses and progression-free survival (PFS) for the above five patients are summarized in Table 1 . The median PFS (mPFS) was 8 months, ranging from 2-12 months. The immunotherapies used were as follows: two patients were treated with sintilimab (Case 1 and Case 2), and three patients were treated with pembrolizumab (Case 3, Case 4 and Case 5). Only one patient (Case 5) had squamous carcinoma, and she was also the only patient with a CR. Figure 1 displays the representative chest CT images of all the patients' best responses.

\section{DISCUSSION}

Five advanced NSCLC patients, three with HER2 mutations and two with HER2 amplifications, received chemoimmunotherapy as the first-line treatment. Among all the patients, the immunotherapies used were as follows: two patients were treated with sintilimab, and three patients were treated with pembrolizumab. Only one patient had squamous carcinoma, and she was also the only patient with a CR. The PFS ranged from 2-12 months, with a median PFS of 8.0 months.

HER2, also known as ERBB2, is a cell surface receptor tyrosine kinase of the ERBB family that is considered an oncogenic driver in many cancers, notably breast, ovarian and gastroesophageal cancers (8). The HER2 receptor is activated via heterodimerization or homodimerization with other ERBB family receptors, inducing activation of EGFR signaling (9). The main types of HER2 alterations in lung cancer include gene insertion mutation, gene amplification and protein overexpression. HER2 insertion mutations and amplifications have been reported in approximately $2-5 \%$ and $2-3 \%$ of lung adenocarcinomas, respectively (10-12).

Recently, many clinical trials have focused on HER2-targeted therapy for HER2-positive NSCLC. However, the results are ambiguous and insufficient. Trastuzumab, a monoclonal antibody for the HER2 receptor, did not show definite benefits for HER2-positive NSCLC patients (13). In contrast, TKIs targeting both HER2 and EGFR were shown to exhibit a therapeutic response. In the EUHER2 study, HER2-targeted drugs, including trastuzumab, lapatinib, neratinib and afatinib, did not show clear survival benefits compared with conventional therapy, including chemotherapy and reversible EGFR-TKIs (7). Among them, afatinib, an irreversible ERBB family blocker, might be a promising therapeutic choice for HER2-mutant NSCLC with progression after previous chemotherapy or reversible EGFR-TKI treatment (14). Afatinib showed a response rate of $18.2 \%$ and an mPFS of 3.9 months in the EUHER2 study (7), but recent phase II trials found that only

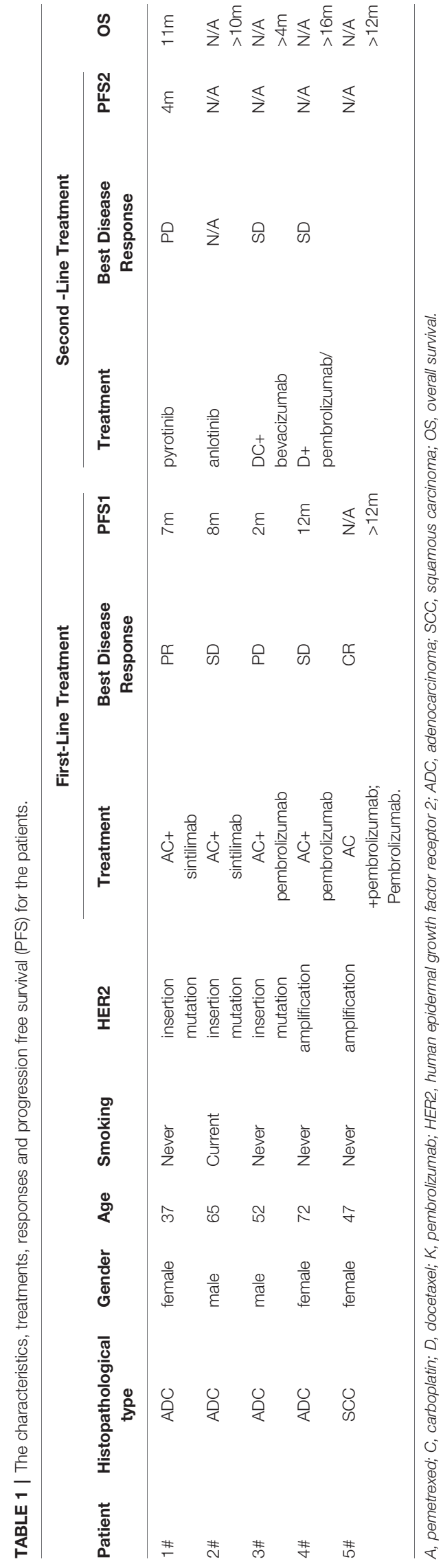



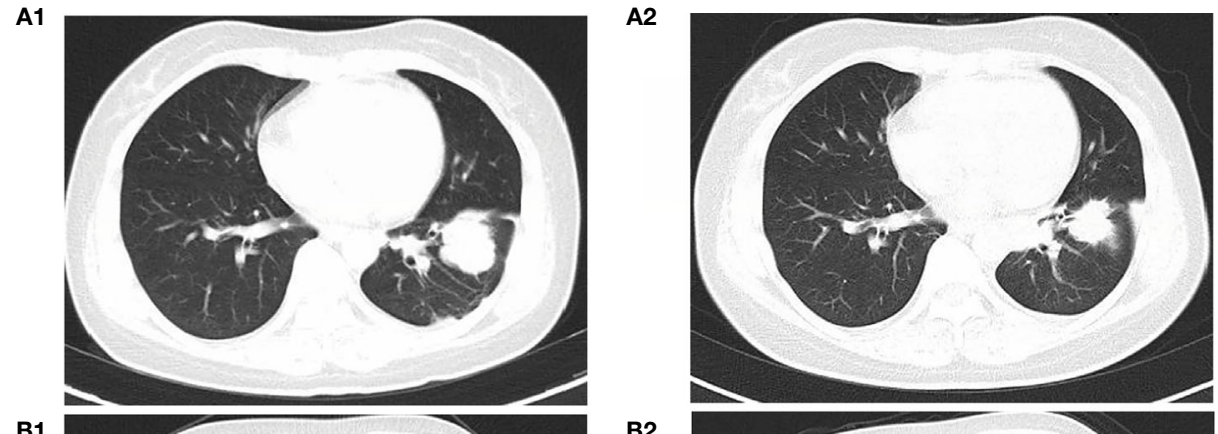

B1

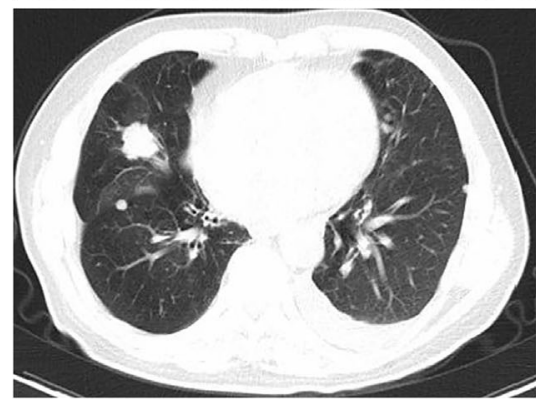

C1

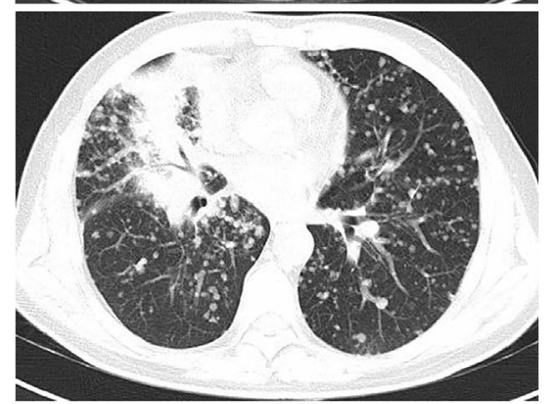

D1

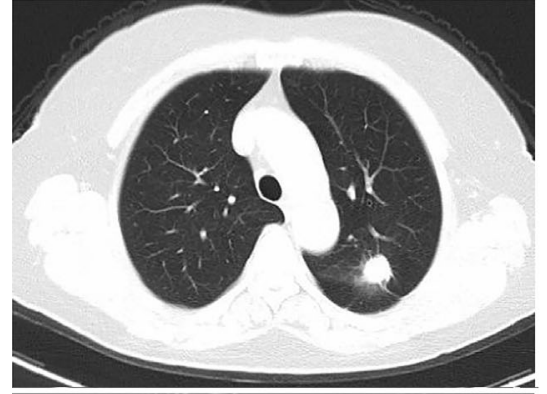

E1

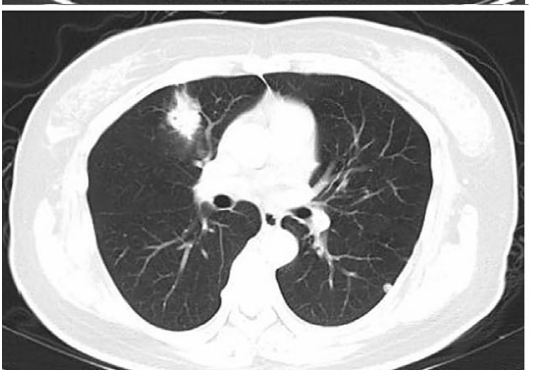

B2

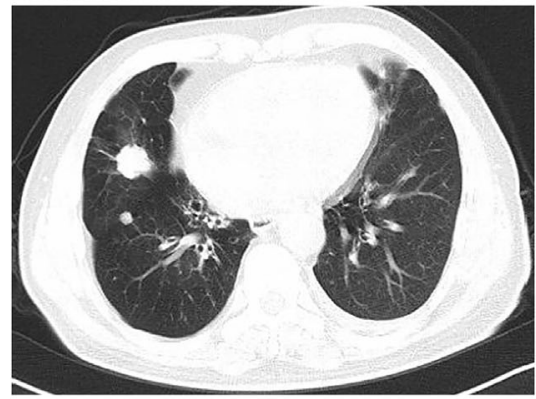

C2

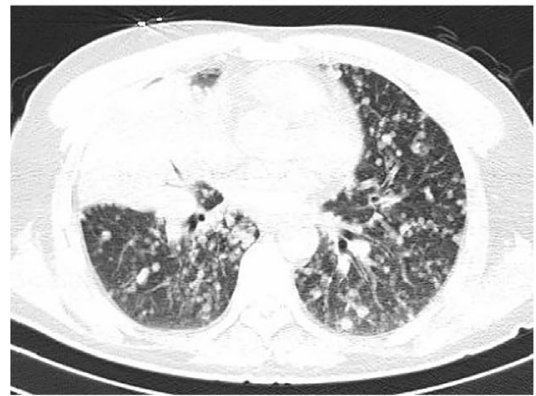

D2

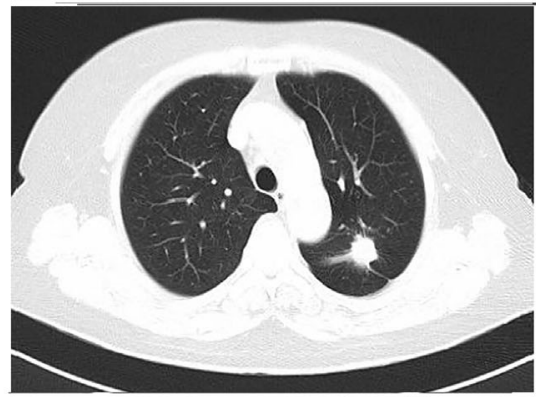

E2

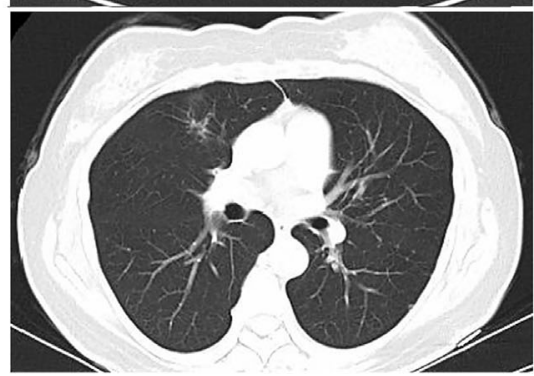

FIGURE 1 | CT imaging of best disease response of patients: Case 1 at beginning of therapy (A1) and 4 months after receiving carboplatin+pemetrexed chemotherapy and sintilimab (A2) Case 2 at beginning of therapy (B1) and 5 months after receiving carboplatin+pemetrexed chemotherapy and sintilimab (B2) Case 3 at beginning of therapy (C1) and 2 months after receiving carboplatin+pemetrexed chemotherapy and pembrolizumab(C2) Case 4 at beginning of therapy (D1) and 10 months after receiving carboplatin+pemetrexed chemotherapy and pembrolizumab (D2) Case 5 at beginning of therapy (E1) and 6 months after treatment with carboplatin, pemetrexed, and pembrolizumab for 4 cycles followed by pembrolizumab maintenance (E2). 
patients with specific HER2 mutations had durable responses to afatinib (15). Subsequently, preliminary results for several other HER2 kinase inhibitors, including temsirolimus (16) and TAK788 (17), have indicated an effect on regression in these patients. Thus far, HER2-targeted therapy has not achieved ideal effects, and the treatment of NSCLC patients with HER2 alterations remains a major challenge.

The advantages of HER2-targeted therapy over chemotherapy in HER2-positive NSCLC are inconclusive. Previous studies suggested that the mPFS durations of chemotherapy alone, pemetrexed \pm platinum/bevacizumab, gemcitabine, taxane \pm platinum/bevacizumab, and vinorelbine were 4.3 months, 6.2 months, 2.6 months, 4 months and 3.5 months, respectively. By comparison, the mPFS of HER2 TKIs was only 2.2 months (18). For HER2-mutant lung cancers, the ORR was 36\%, and the mPFS was 5.1 months with chemotherapy as the first-line therapy (6). The ORR and mPFS were $50.9 \%$ and 4.8 months, respectively, with trastuzumab or ado-trastuzumab emtansine (T-DM1) (7). Therefore, chemotherapy remains an important component of treatment, while the benefit of HER2-targeted therapy is inconclusive. However, the outcome of NSCLC patients with HER2 alterations who are treated with chemotherapy can be further improved by combination treatment, and thus, additional therapies for these patients are warranted.

Immunotherapies are also worth considering for the treatment of patients with HER2 alterations. The combination treatment of pembrolizumab and chemotherapy was included in the guidelines as a first-line treatment for advanced NSCLC based on the KEYNOTE-189 trial $(19,20)$. Nevertheless, immunotherapy is less effective in patients with oncogenic mutations than in patients without oncogenic mutations, and anti-PD-1/PD-L1 therapy may even facilitate hyperprogression (21). Chiara Catania et al. reported a case in which nivolumab had strong antitumor activity in advanced HER2-positive lung cancer (22). Conversely, Jody C. Chuang reported that HER2mutated NSCLC patients did not respond to nivolumab (23). Mazieres et al. reported an ORR of 7\% and a median PFS of 2.5 months amongst 29 patients with HER2 altered advanced lung cancer when treated with single agent immune checkpoint inhibitors (24). In our study, five advanced NSCLC cases were described. The results showed that the PFS times of the patients ranged from 2-12 months, with an mPFS of 8.0 months with chemoimmunotherapy. Based on our experience, we propose that chemoimmunotherapy may be a hopeful first-line treatment option for NSCLC patients with HER2 alterations.

NSCLC has distinct clinical features according to the HER2 alteration type; however, both amplification and oncogenic mutation in HER2 can promote receptor hyperactivation and tumor growth (25). HER2 mutations mainly occur at exon 20 in the protein kinase domain and are recognized as primary drivers in lung cancer, similar to other oncogenic drivers, such as EGFR, ROS, ALK, KRAS and BRAF (11). In NSCLC, it is controversial whether HER2 amplification is a driver gene. Some studies have suggested that amplification of ERBB2 is a driver event specifically in oncogene-negative lung adenocarcinoma (12). However, HER2 amplification may not be associated with HER2 mutation, and they may be involved in distinct clinical entities that need different therapeutic methods (26). Case reports have suggested that pyrotinib and afatinib may also be effective for lung adenocarcinoma patients with coexisting HER2 mutation and amplification $(27,28)$. The anti-HER2 antibody-drug conjugates (ADCs) have shown greater clinical benefit than TKIs in HER2amplified cancers (29), specifically T-DM1 and deruxtecantrastuzumab (T-DXd). T-DM1 was clinically effective in ERBB2amplified/mutant lung cancer patients, and the ORR was 51\%, with a mPFS of 5 months $(30,31)$. For heavily pretreated HER2mutant NSCLC, a phase I study showed that the ORR of T-DXd was $72.7 \%$, and the median PFS was 11.3 months (95\% CI, 8.114.3) (32). Then in an ongoing phase II study (DESTINY-Lung01 study), T-DXd demonstrated an encouraging efficacy in this molecular subset of lung cancers. The ORR was $61.9 \%$, and the median PFS was 14.0 months (95\% CI, 6.4-14.0) (33). However, all of these studies are still in phase I or II, and the sample size is relatively small. Therefore, further exploration is required to identify specific types of HER2 alterations and assess their potential as novel therapeutic targets.

There are several limitations to this study. First, the sample size was too small, and the statistical results may have biased. Therefore, more prospective, larger sample size, randomized, controlled studies are needed. Second, the PD-L1 expression of these patients was negative, so the impact of PD-L1 status on the treatment response in these patients is unknown. Finally, the follow-up time of our study was only 12 months, and the overall survival (OS) of most patients was not reached. Thus, the patients still need to be followed up. Thus, the evidence from current clinical practice is inadequate, and further clinical data are needed to confirm our results.

To our knowledge, this study is the first to report chemoimmunotherapy as a first-line treatment for advanced NSCLC with HER2 alterations. Overall, our study aimed to provide additional data regarding the treatment of NSCLC with HER2 mutation or amplification. The results suggest that chemoimmunotherapy may be a hopeful first-line treatment option for these patients. However, further clinical trials are required to expand treatment options for NSCLC patients with HER2 alterations.

\section{DATA AVAILABILITY STATEMENT}

The original contributions presented in the study are included in the article/supplementary material. Further inquiries can be directed to the corresponding authors.

\section{ETHICS STATEMENT}

The studies involving human participants were reviewed and approved by The Committee on Medical Ethics of West China Hospital. The patients/participants provided their written informed consent to participate in this study. Written 
informed consent was obtained from the individual(s) for the publication of any potentially identifiable images or data included in this article.

\section{AUTHOR CONTRIBUTIONS}

SZ wrote the manuscript. SZ and XX collected the data. SZ and PT analyzed the data. KW and YL revised the manuscript. KW and WL designed the manuscript. KW and YL reviewed the

\section{REFERENCES}

1. Siegel RL, Miller KD, Jemal A. Cancer statistics, 2020. CA Cancer J Clin (2020) 70:7-30. doi: 10.3322/caac. 21590

2. Majem M, Juan O, Insa A, Reguart N, Trigo JM, Carcereny E, et al. SEOM clinical guidelines for the treatment of non-small cell lung cancer (2018). Clin Transl Oncol (2019) 21:3-17. doi: 10.1007/s12094-018-1978-1

3. Campbell JD, Alexandrov A, Kim J, Wala J, Berger AH, Pedamallu CS, et al. Distinct patterns of somatic genome alterations in lung adenocarcinomas and squamous cell carcinomas. Nat Genet (2016) 48:607-16. doi: 10.1038/ng.3564

4. Peters S, Zimmermann S. Targeted therapy in NSCLC driven by HER2 insertions. Transl Lung Cancer Res (2014) 3:84-8. doi: 10.3978/j.issn.22186751.2014.02.06

5. Kuyama S, Hotta K, Tabata M, Segawa Y, Fujiwara Y, Takigawa N, et al. Impact of HER2 gene and protein status on the treatment outcome of cisplatin-based chemoradiotherapy for locally advanced non-small cell lung cancer. J Thorac Oncol (2008) 3:477-82. doi: 10.1097/JTO.0b013e31816e2ea3

6. Wang Y, Zhang S, Wu F, Zhao J, Li X, Zhao C, et al. Outcomes of Pemetrexedbased chemotherapies in HER2-mutant lung cancers. BMC Cancer (2018) 18:326. doi: 10.1186/s12885-018-4277-x

7. Mazières J, Barlesi F, Filleron T, Besse B, Monnet I, Beau-Faller M, et al. Lung cancer patients with HER2 mutations treated with chemotherapy and HER2targeted drugs: results from the European EUHER2 cohort. Ann Oncol (2016) 27:281-6. doi: 10.1093/annonc/mdv573

8. Baselga J, Swain SM. Novel anticancer targets: revisiting ERBB2 and discovering ERBB3. Nat Rev Cancer (2009) 9:463-75. doi: 10.1038/nrc2656

9. Oxnard GR, Binder A, Jänne PA. New targetable oncogenes in non-small-cell lung cancer. J Clin Oncol (2013) 31:1097-104. doi: 10.1200/JCO.2012.42.9829

10. Arcila ME, Chaft JE, Nafa K, Roy-Chowdhuri S, Lau C, Zaidinski M, et al. Prevalence, clinicopathologic associations, and molecular spectrum of ERBB2 (HER2) tyrosine kinase mutations in lung adenocarcinomas. Clin Cancer Res (2012) 18:4910-8. doi: 10.1158/1078-0432.CCR-12-0912

11. Kris MG, Johnson BE, Berry LD, Kwiatkowski DJ, Iafrate AJ, Wistuba II, et al. Using multiplexed assays of oncogenic drivers in lung cancers to select targeted drugs. JAMA (2014) 311:1998-2006. doi: 10.1001/jama.2014.3741

12. Cancer Genome Atlas Research Network. Comprehensive molecular profiling of lung adenocarcinoma. Nature (2014) 511:543-50. doi: 10.1038/nature13385

13. Mar N, Vredenburgh JJ, Wasser JS. Targeting HER2 in the treatment of nonsmall cell lung cancer. Lung Cancer (2015) 87:220-5. doi: 10.1016/ j.lungcan.2014.12.018

14. Oh IJ, Hur JY, Park CK, Kim YC, Kim SJ, Lee MK, et al. Clinical Activity of Pan-HER Inhibitors Against HER2-Mutant Lung Adenocarcinoma. Clin Lung Cancer (2018) 19:e775-81. doi: 10.1016/j.cllc.2018.05.018

15. Kris MG, Camidge DR, Giaccone G, Hida T, Li BT, O'Connell J, et al. Targeting HER2 aberrations as actionable drivers in lung cancers: phase II trial of the pan-HER tyrosine kinase inhibitor dacomitinib in patients with HER2-mutant or amplified tumors. Ann Oncol (2015) 26(7):1421-7. doi: 10.1093/annonc/mdv186

16. Besse B, Soria J-C, Yao B, Kris M, Chao B, Cortot A, et al. Neratinib (N) with or Without Temsirolimus (Tem) in Patients (Pts) with Non-Small Cell Lung Cancer (Nsclc) Carrying Her2 Somatic Mutations: an International Randomized Phase II Study. Ann Oncol (2014) 25(suppl 4):v1-v41. doi: 10.1093/annonc/mdu438.47 manuscript. All authors contributed to the article and approved the submitted version.

\section{FUNDING}

This work was supported by grants from National Natural Science Foundation of China (Grants 82070019 and 81870034) and Sichuan Science and Technology Program (No. 2020YFS0572).

17. Janne PA, Neal JW, Camidge DR, Spira AI, Piotrowska Z, Horn L, et al. Antitumor activity of TAK-788 in NSCLC with EGFR exon 20 insertions. J Clin Oncol (2019) 37 May (15_suppl):9007. doi: 10.1200/JCO.2019. 37.15_suppl.9007

18. Eng J, Hsu M, Chaft JE, Kris MG, Arcila ME, Li BT. Outcomes of chemotherapies and HER2 directed therapies in advanced HER2-mutant lung cancers. Lung Cancer (2016) 99:53-6. doi: 10.1016/j.lungcan.2016. 05.030

19. Gadgeel S, Rodríguez-Abreu D, Speranza G, Esteban E, Felip E, Dómine M, et al. Updated Analysis From KEYNOTE-189: Pembrolizumab or placebo plus pemetrexed and platinum for previously untreated metastatic nonsquamous non-small-cell lung cancer. J Clin Oncol (2020) 38:1505-17. doi: 10.1200/JCO.19.03136

20. Gandhi L, Rodríguez-Abreu D, Gadgeel S, Esteban E, Felip E, De Angelis F, et al. KEYNOTE-189 Investigators. Pembrolizumab plus Chemotherapy in Metastatic Non-Small-Cell Lung Cancer. N Engl J Med (2018) 37:2078-92. doi: 10.1056/NEJMoa1801005

21. Pardoll DM. The blockade of immune checkpoints in cancer immunotherapy. Nat Rev Cancer (2012) 12:252-64. doi: 10.1038/nrc3239

22. Catania C, Passaro A, Rocco EG, Spitaleri G, Barberis M, Noberasco C, et al. Dramatic Antitumor Activity of Nivolumab in Advanced HER2-Positive Lung Cancer. Clin Lung Cancer (2016) 17:e179-83. doi: 10.1016/ j.cllc.2016.05.004

23. Chuang JC, Stehr H, Liang Y, Das M, Huang J, Diehn M, et al. ERBB2Mutated Metastatic Non-Small Cell Lung Cancer: Response and Resistance to Targeted Therapies. J Thorac Oncol (2017) 12:833-42. doi: 10.1016/ j.jtho.2017.01.023

24. Mazieres J, Drilon A, Lusque A, Mhanna L, Cortot AB, Mezquita L, et al. Immune checkpoint inhibitors for patients with advanced lung cancer and oncogenic driver alterations: results from the IMMUNOTARGET registry. Ann Oncol (2019) 30(8):1321-8. doi: 10.1093/annonc/mdz167

25. Yarden Y, Sliwkowski MX. Untangling the ErbB signalling network. Nat Rev Mol Cell Biol (2001) 2:127-37. doi: 10.1038/35052073

26. Li BT, Ross DS, Aisner DL, Chaft JE, Hsu M, Kako SL, et al. HER2 Amplification and HER2 Mutation Are Distinct Molecular Targets in Lung Cancers. J Thorac Oncol (2016) 1:414-9. doi: 10.1016/j.jtho.2015.10.025

27. Shan J, Ruan J, Tan Y, Yan L, Chen S, Du M, et al. Efficacy of Pyrotinib in a Heavily Pretreated Patient with Lung Adenocarcinoma Harboring HER2 Amplification and Exon 20 Insertions: A Case Report. Oncol Targets Ther (2020) 13:9849-56. doi: 10.2147/OTT.S271999

28. Liu X, Cao Y, Li Y, Duan X. Advanced lung adenocarcinoma with coexistent HER2 mutation and amplification and response to afatinib: a case report. Ann Palliat Med (2020) 9(2):483-7. doi: 10.21037/apm.2020.02.14

29. Moasser MM. Two dimensions in targeting HER2. J Clin Oncol (2014) 32:2074-7. doi: 10.1200/JCO.2014.55.7652

30. Li BT, Michelini F, Misale S, Cocco E, Baldino L, Cai Y, et al. HER2-Mediated Internalization of Cytotoxic Agents in ERBB2 Amplified or Mutant Lung Cancers. Cancer Discovery (2020) 10:674-87. doi: 10.1158/2159-8290.CD-200215

31. Li BT, Shen R, Buonocore D, Olah ZT, Ni A, Ginsberg MS, et al. Adotrastuzumab emtansine for patients with HER2-mutant lung cancers: results 
from a phase II bsket trial. J Clin Oncol (2018) 36(24):2532-7. doi: 10.1200/ JCO.2018.77.9777

32. Tsurutani J, Iwata H, Krop I, Jänne PA, Doi T, Takahashi S, et al. Targeting HER2 with Trastuzumab Deruxtecan: A Dose-Expansion, Phase I Study in Multiple Advanced Solid Tumors. Cancer Discovery (2020) 10(5):688-701. doi: 10.1158/2159-8290.CD-19-1014

33. Egbert FS, Kazuhiko N, Misako N, Enriqueta F, Yasushi G, Li BT, et al. Trastuzumab deruxtecan (T-DXd; DS-8201) in patients with HER2-mutated metastatic non-small cell lung cancer (NSCLC): Interim results of DESTINYLung01. J Clin Oncol (2020) 31(suppl 3):235. doi: 10.1200/jco.2020. 38.15_suppl.9504
Conflict of Interest: The authors declare that the research was conducted in the absence of any commercial or financial relationships that could be construed as a potential conflict of interest.

Copyright $\odot 2021$ Zhao, Xian, Tian, Li, Wang and Li. This is an open-access article distributed under the terms of the Creative Commons Attribution License (CC BY).

The use, distribution or reproduction in other forums is permitted, provided the original author(s) and the copyright owner(s) are credited and that the original publication in this journal is cited, in accordance with accepted academic practice. No use, distribution or reproduction is permitted which does not comply with these terms. 